\title{
Peptide Surrogates and Antioxidants in the Combat Against Neurodegeneration
}

\section{Shimon Shatzmiller*, Inbal Lapidot, Galina Zats and Ludmila Buzhansky}

Department of Chemical Sciences, Ariel University, Ariel, Israel

*Corresponding Author: Shimon Shatzmiller, Department of Chemical Sciences, Ariel University, Ariel, Israel.

Received: April 30, 2019; Published: July 09, 2019

DOI: 10.31080/ASNE.2019.02.0077

\begin{abstract}
The quest of remedy for neurodegenerative diseases is already more than a century old. It is unclear, applying the moist sophisticated ultramodern methods and equipment, what is in fact going on in the human brain when this curse hits. However, Nowadays the perception is that gut microbiome penetrates the brain via the "Gut-Brain" axis. Either by using nerves or through the bloodstream by moving from a leaky gut to a damaged blood-brain barrier. And when in the brain, causing inflammation and subsequent infection, that moves in the brain in a prion-like a mechanism, from one cell to its neighbor until interoception (death) is reached.

Keywords: Peptide; Antioxidants; Neurodegeneration
\end{abstract}

The eradication of bacteria bu antimicrobial peptide surrogates in synergy with antioxidants, substances that comes from nu- trients, to combat inflammation and the crawling infection might be a proper approach to treat neurodegeneration at its early stages.

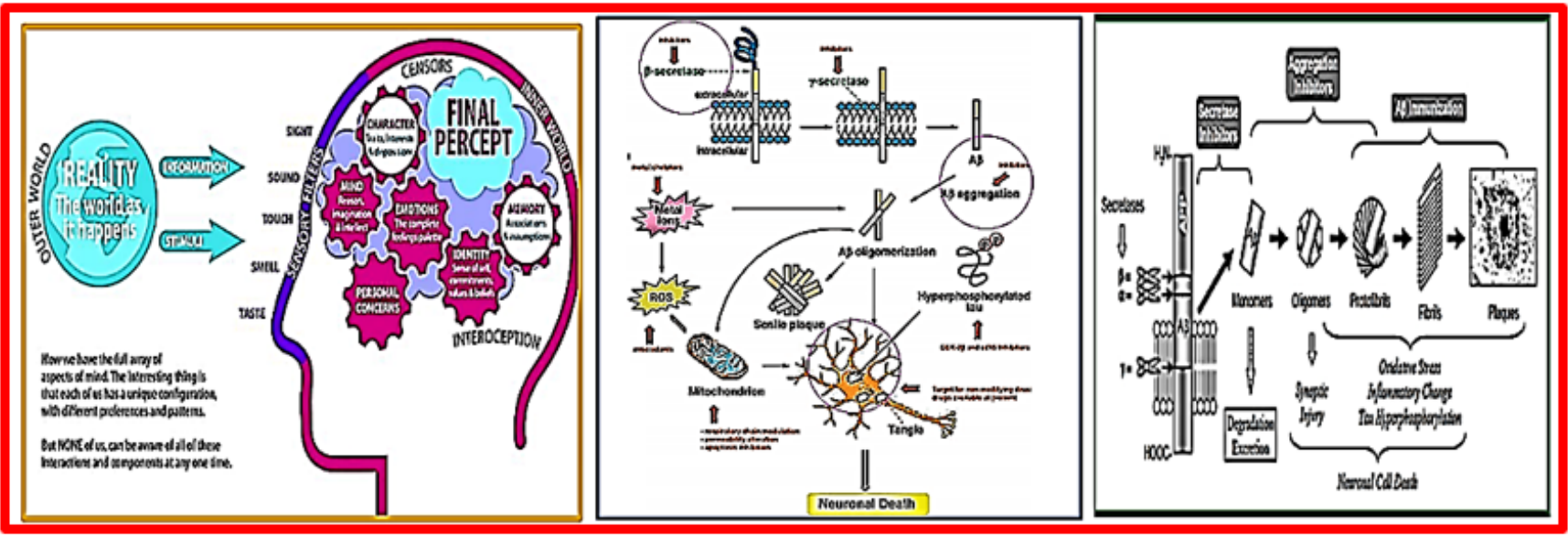

Figure a: The Progression of Neurodegeneration.

The top 10 causes of death, the World Health Organization report [1-7].

Biology of Ageing and Role of Dietary Antioxidants is a leading avenue of research in the area on nerology. It focuses on oxidative stress that is caused by inflammation as well as infection [8].
Tea Catechins and Theaflavins. Tea, next to the water, is the second most popular beverage consumed by humans in the world. Black tea is more widely consumed in Western countries while green tea is preferred in the Eastern world. Black tea extracts mainly contain catechins and theaflavins (Figure). Evidence from clinical trials suggests that the consumption of tea has various health be- 
nefits. Scientists demonstrated that drinking either green tea or black tea would lead to a significant increase in plasma antioxidant potential by ferric-reducing antioxidant power (FRAP) assay.
Furthermore, it has been reported in different population studies that the consumption of green tea or black tea could significantly reduce DNA oxidation and lipid peroxidation.
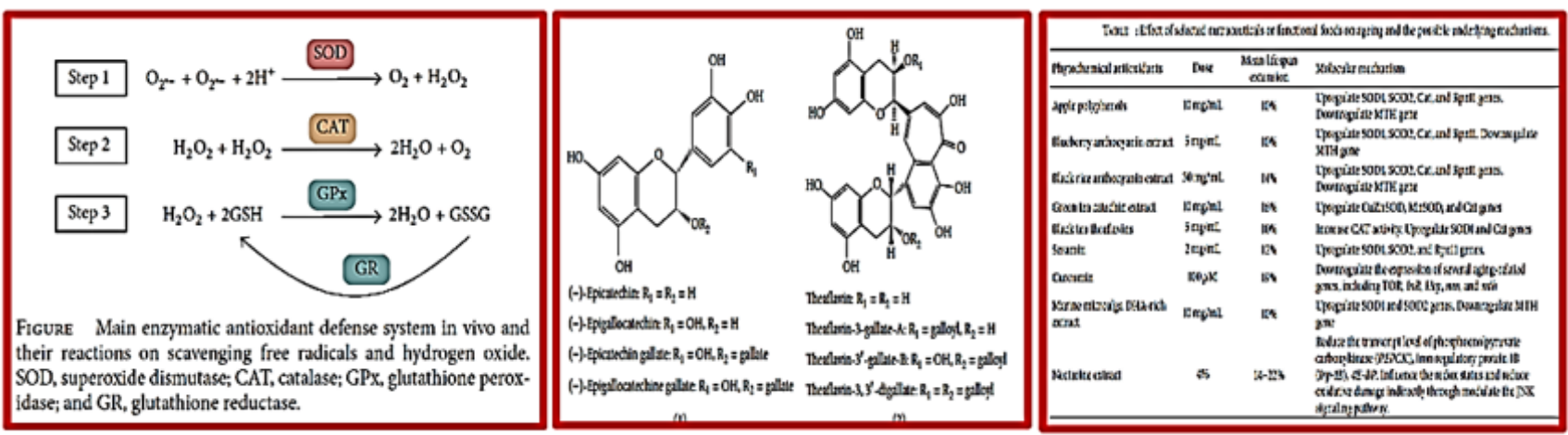

Figure b: Antioxidants of Green Tea (credit ref. [2]).

Forward

Neurodegenerative disorders sudsch as Alzheimer's disease (AD), stroke, and Parkinson's disease (PD) represent a major clinical problem in the developed countries [9] and are major economic burdens for health care systems. Dietary [10], genetic, and molecular factors [11] are important determinants in the progression and intervention of neurodegenerative diseases. AD is a common cause of dementia and mortality in the United States. Total numbers of reported deaths due to AD have increased in the past years, and it is among 10 leading causes of deaths in the western world $[12,13]$. Recent work has demonstrated that the microbiota also influences brain function in healthy and diseased individuals. Of great interest are reports that intestinal bacteria play a role in the pathogenic cascade of both Parkinson and Alzheimer diseases. Prion-like mechanisms [14] in neurodegenerative diseases are causing great difficulty in an attempt to stop the spread of the disease in the brain (Figure c).

If trans-cellular propagation of protein misfolding occurs, then new strategies could supplement existing approaches to promote cell survival and block the intracellular accumulation of misfolded species. As the cellular mechanisms of aggregate release and uptake are delineated, it may be possible to inhibit these events pharmacologically or genetically. Antibody-based therapies might also be expanded to target protein aggregates that are generated inside a cell and released into the extracellular space (Figure d).

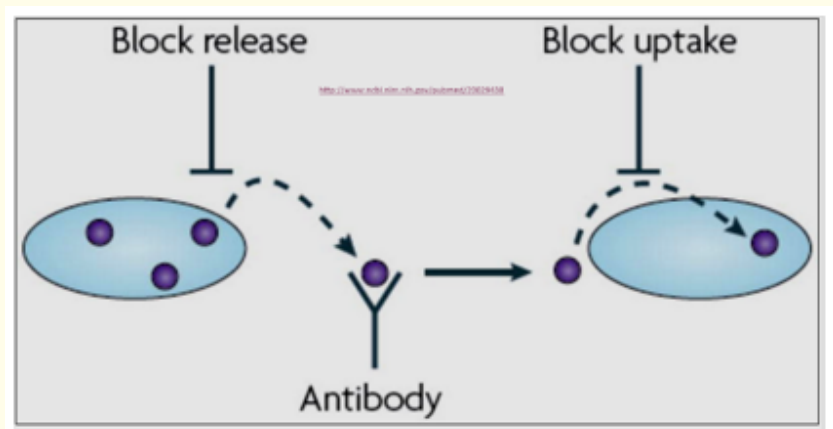

Figure c

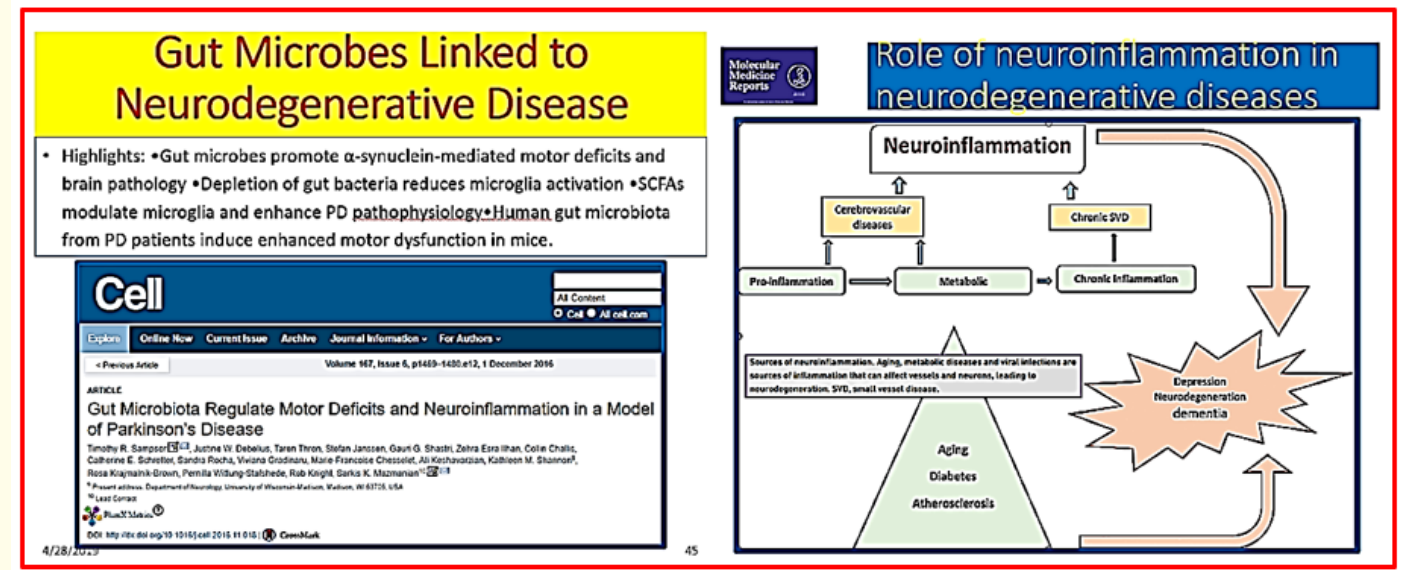

Figure d 
It is believed that "Eating the rainbow" [15-17] is a modern slogan by nutrition experts. This "Mediterranean" food intake is believed by many to bring some remedy to the many millions worldwide that are victims of neurodegenerative diseases, like Alzheimer's, Parkinson's, Brain Stroke and many other. This diet will provide active chemicals produced by nature to combat some factors that initiate and keep the illnesses progressing, slowly but surely. According to a recently applied conception, neurodegeneration may be a cause of penetration of gut microbiome [18] bacteria through a leaky gut and a damaged Blood-Brain Barrier [19-21] infiltrate the barrier and cause inflammation and subsequent crawling infection, in the brain which ultimately brings the patient to death.

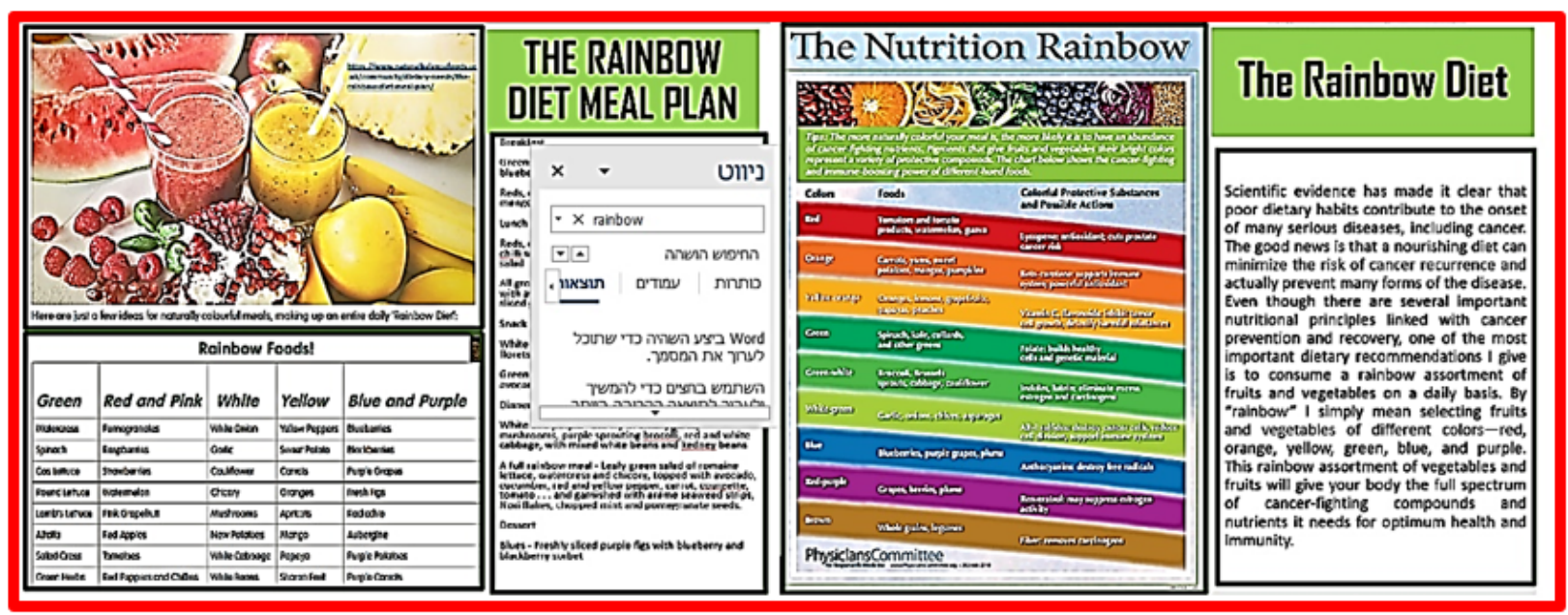

Figure e

The onset of neurodegeneration is believed to involve oxidative stress due to the invasion of the gut microbiome into the brain tissue which causes inflammation, marked by oxidative stress.

A synergy between antimicrobial and antioxidant agents may address these evolvements.
The antimicrobial part maybe based on antimicrobial peptide surrogate and the antioxidant portion may involve polyphenols $[22,23]$ as antioxidants penetrating the brain by nutrition, consuming foods that carry such compounds in its matter.

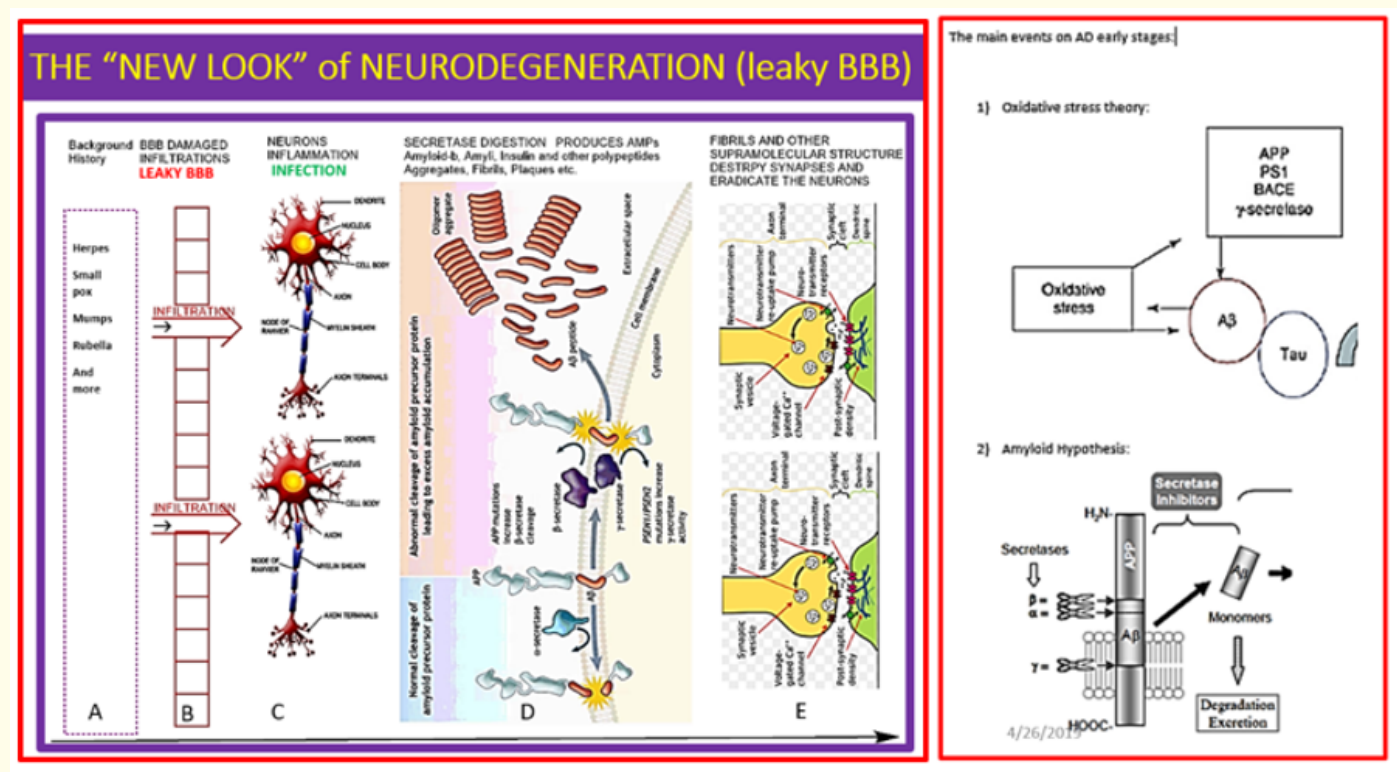

Figure $\mathbf{f}$

Citation: Shimon Shatzmiller., et al. "Peptide Surrogates and Antioxidants in the Combat Against Neurodegeneration". Acta Scientific Neurology 2.8 (2019): 26-37. 
The gut-brain microbiome axis has received an abundance of attention recently. It is thought that gut microbiota can influence brain function and behavior, but how that happens is still unknown. It has been proposed that bacteria can enter the brain through the blood-brain barrier, and/or via nerves that innervate the gut. Here we show the presence of bacteria in the human and mouse brain under noninfectious or nontraumatic conditions. It was found the bacteria, identified by morphological criteria, in ultrastructural samples of human postmortem brain. Serial section analysis for identification and quantification. All cases contained bacteria in varying amounts. Bacteria were rod-shaped, and contained a capsule, nucleoid, ribosomes and vacuoles. The average diameter of the short axis was $0.496 \mathrm{~mm}$. Many were segmented, with the long axis averaging approximately $1.78 \mathrm{~mm}$ between segments. Others did not appear to be segmented and were approximately.

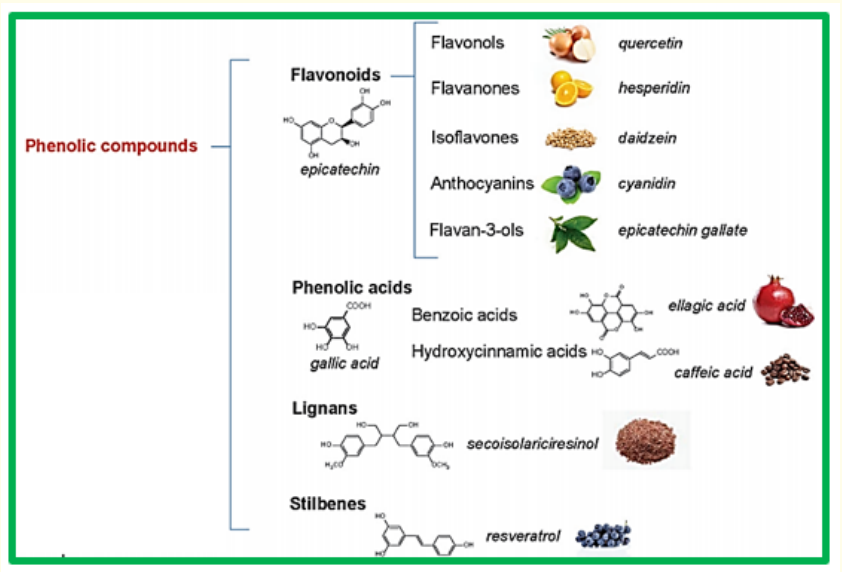

Figure g: Phenolic compounds in "Mediterranean Diet" (credit ref. 8).

$0.866 \mathrm{~mm}$ in the long axis. The vast majority of the profiles had a thick capsule of approximately $100 \mathrm{~nm}$. The density of the bacteria varied according to the brain region, with abundant bacteria in the substantia nigra, hippocampus and prefrontal cortex but sparse numbers in the striatum. Bacteria were present in intracellular locations, predominantly in astrocytic end feet at the blood-brain barrier, dendrites and the soma of glial cells. They were also abundant adjacent to and within myelinated axons. To address the possibility that the bacteria in human tissue was a result of postmortem artifact, we examined mouse brains that were fixed immediately at death $(n=10)$; there were abundant bacteria in similar intracellular locations. To eliminate the possibility that the presence of bacteria was due to contamination, we examined germ-free mouse brains $(n=4)$ processed in an identical way; we did not detect any bacteria. The observation that the location of the bacteria was highly specific and deep within the specimens also argues against contamination. Interestingly, there were no structural signs of inflammation in any of the brains examined. It is presently unclear the route of entry bacteria take to the brain, but the evidence of them in axons and at the blood-brain barrier supports the previous speculation.

Alpha-synuclein $(\alpha S)$ is a nerve cell protein associated with Parkinson disease (PD) [24]. Accumulation of $\alpha \mathrm{S}$ within the enteric nervous system (ENS) and its traffic from the gut to the brain are implicated in the pathogenesis and progression of PD. $\alpha \mathrm{S}$ has no known function in humans and the reason for its accumulation within the ENS is unknown. Several recent studies conducted in rodents have linked $\alpha \mathrm{S}$ to immune cell activation in the central nervous system. We hypothesized that $\alpha \mathrm{S}$ in the ENS might play a role in the innate immune defenses of the human gastrointestinal (GI) tract.

Our brain is home for microbiome dwellers

Treatment of many neuronal degenerative disorders will require delivery of a therapeutic protein to neurons or glial cells across the whole CNS. The presence of the blood-brain barrier hampers the delivery of these proteins from the blood, thus necessitating a new method for delivery.

Antimicrobial peptides (AMPs) are host-encoded antibiotics that combat invading microorganisms. These short, cationic peptides have been implicated in many biological processes, primarily involving innate immunity.

The penetration of microbes into the inner brain can be the onset of inflammation followed by infection in the brain. One can rationalize the digestion of APP and other proteins by enzymes to form antimicrobial peptides like The amyloid $b$ which combat the microbes, but aggregate to form many sorts of solid formations that damage the synapses as depicted above. The series of events that are induced by the oxidative stress of the inflammation, lead to the harming antimicrobial peptides of which the amyloids are only one sort. Since peptides can barely enter into the brain via the BBB [25]. However, it is possible to enter the brain via various receptors, an example is the diazepine receptor [26].

In innate immunity, inflammation-based immunity is the first line of vertebrate defense against micro-organisms. Inflammation relies on a number of cellular and molecular effectors that can strike invading pathogens very shortly after the encounter between inflammatory cells and the intruder, but in a non-specific way. 
Owing to this non-specific response, inflammation can generate substantial costs for the host if the inflammatory response, and the associated oxygen-based damage, get out of control. This imposes strong selection pressure that acts to optimize two key features of the inflammatory response: the timing of activation and resolution (the process of downregulation of the response). Nevertheless, host immune regulation also opens the way to pathogens to subvert host defenses.

Currently, many agree that the gut microbiome reaches the brain using thew "gut-brain axis" and is the cause for the onset of neurodegeneration - this penetration results in inflammation, then infection and apoptosis of the brain tissue. Antimicrobial peptide surrogates [27], based on a brain-penetrating scaffold-like diazepine [28] or 1,4 dihydropyridine [29] may be suitable to combat the invading microbiota and in a later stage serve as antimicrobial agents to combat the infection and prevent aggregation.

Blueberries aren't actually blue [30], but deep purple, which is the color of anthocyanin, a the pigment that is especially rich in blueberries $[32,33]$. Blueberries are known to be high in antioxidants, "Blueberries are the King of Antioxidant Foods", which are good for the human body; the anthocyanin is thought to be useful for combating inflammation. A good rule to follow is, the darker the berry, the more anthocyanins are present. The main antioxidant compounds in blueberries belong to a family of polyphenols antioxidants called flavonoids.

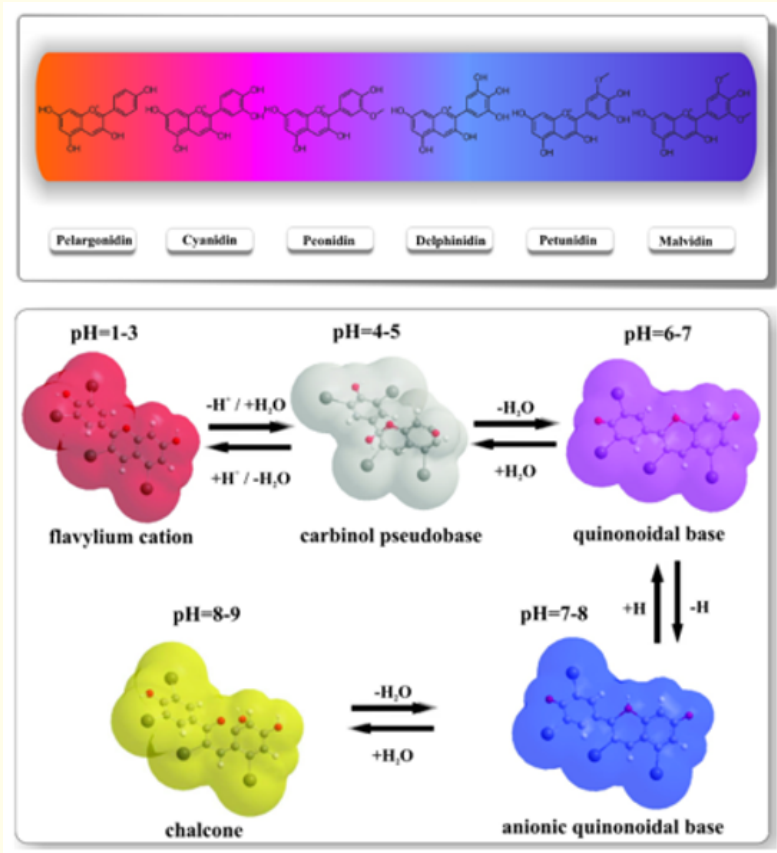

Figure h: The visible color range of common anthocyanidins (credit ref. [31]).
The group of flavonoids in particular — anthocyanins - is thought to be responsible for much of these berries' beneficial health effects.

There are many antioxidants that may aid in this circumstance for an instant in a Mediterranean diet or by using forest berries, blueberries [34] that contain many sorts of antioxidants. In fact, blueberry intake acutely improves vascular function in healthy men in a time- and intake-dependent manner. These benefits may be mechanistically linked to the actions of circulating phenolic metabolites on neutrophil NADPH oxidase activities. Identification of Phenolic Acids, Flavonol Glycosides and Antioxidant Potential in Blueberry, Blackberry, Raspberries and Cranberries were reported [35].

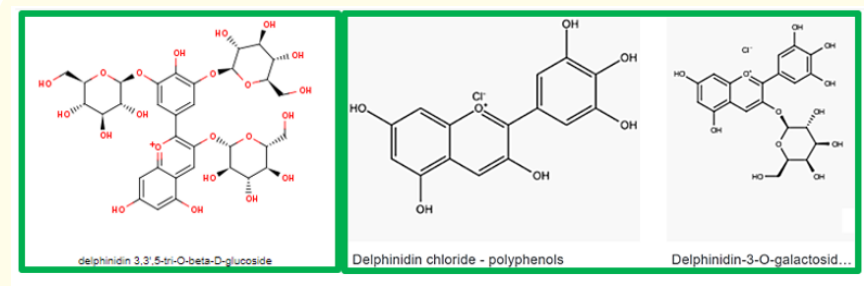

Figure i

The daily consumption of fruits and vegetables is consistently associated with reductions in the incidence of degenerative diseases, by having antioxidant (The blue pigment Delphinidin for example. Delphinidin is the most common anthocyanidin molecule in blue flowers and forms with pelargonidin and cyanidin the three anthocyanidins known as the principal and basic skeletons of flower color pigments. so they are the most widespread in nature), anti-inflammatory and anticarcinogenic activity [36]. Scientists reported [37], that daily consumption of $600 \mathrm{~g}$ of fruits or vegetables can prevent the risk of coronary heart disease by more than $31 \%$ and ischemic stroke by $19 \%$. Several epidemiological studies summarized in diverse papers [38-41] have built the consensus that diets rich in fruits and vegetables have beneficial effects on human health (Figure j).

Scientists at the University of Basel $[42,43]$ discovered a fundamentally new mechanism explaining the inadequate immune defense against chronic viral infection.

Hope for remedy?

Crossing the BBB is a major obstacle in an attempt to find a therapy for neurodegeneration. Thew brain is protected in many ways, by the bones of the skull, and by many layers od different tissues and finally by the blood-brain barrier, which only selected compounds may cross in both directions using various crossing gates [44] (Figure k). 

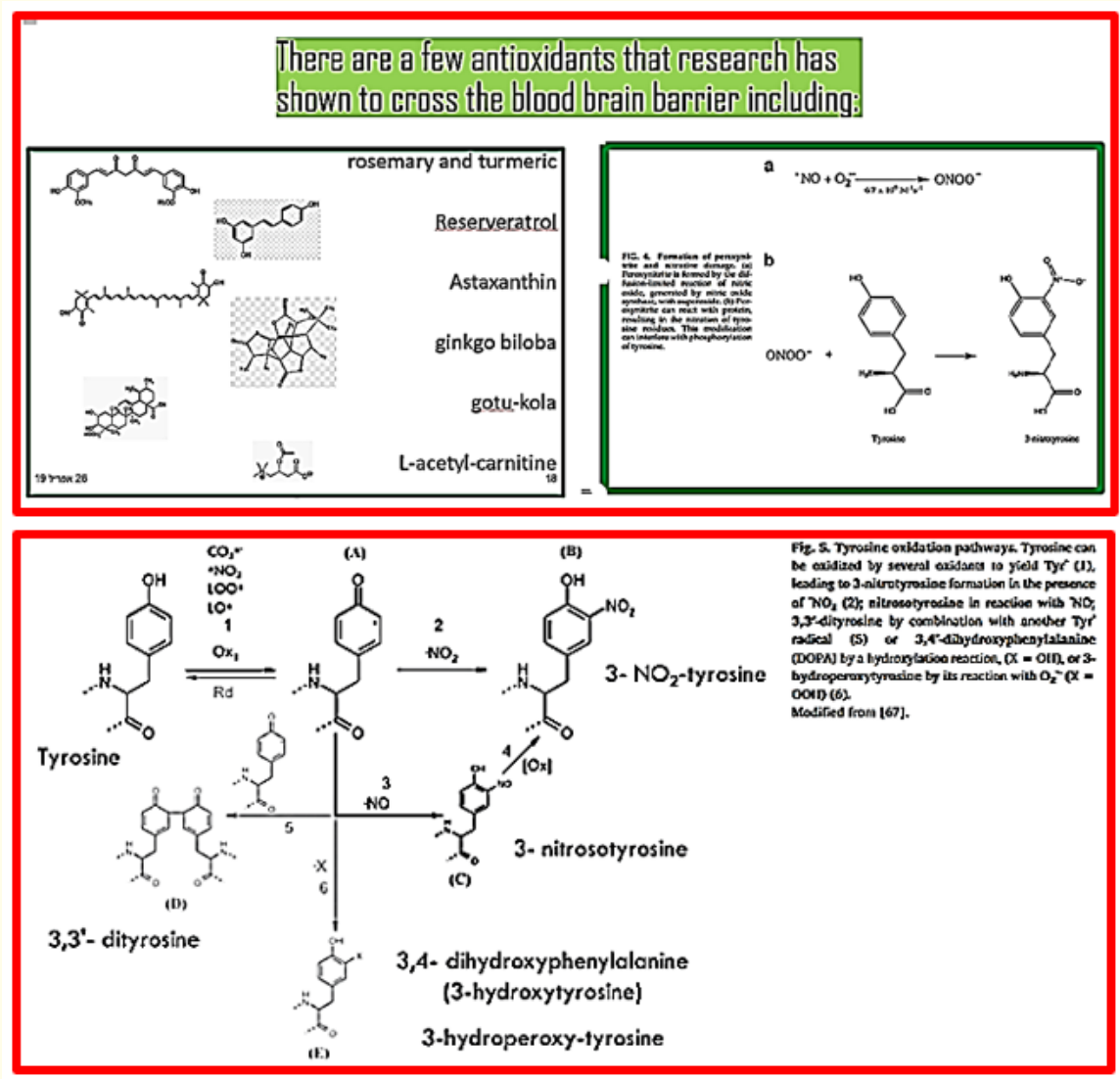

Figure j: Antioxidants that are known to cross into the brain and capture of NOX by tyrosine (Credit ref. [30]).

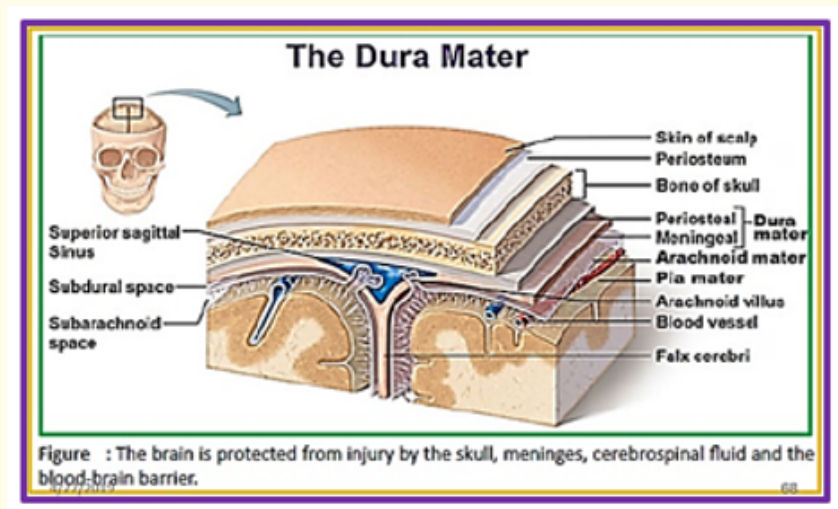

Figure k
The bidirectional crossing of the BBB

The brain is a protected environment, partially walled off from the contents of the bloodstream by a network of cells that surround its blood vessels. Bacteria and viruses that manage to penetrate this blood-brain barrier can cause life-threatening inflammation.

\section{Penetration into brain and CSF}

- The capillary endothelial cells in brain have tight junctions and lack large paracellular spaces. Further, an investment of neural tissue covers the capillaries. Together they constitute the so called blood-brain barrier (BBB). A similar blood-CSF barrier is located in the choroid Plexus.

- Only lipid-soluble drugs are able to penetrate and have action on the central nervous system. $\quad$ T'm somy, but you ar

- Inflammation of meninges or brain increases the risk that some drugs accumulate in the brain. too highly charged, too large and not lipid Hey! W

Some research has suggested distant microbes-those living in our gut - might affect mood and behavior and even the risk of neurological disease, but by indirect means. For example, a disruption in the balance of gut microbiomes could increase the production of a rogue protein that may cause Parkinson's disease if it travels up the nerve connecting the gut to the brain $[45,46]$. 


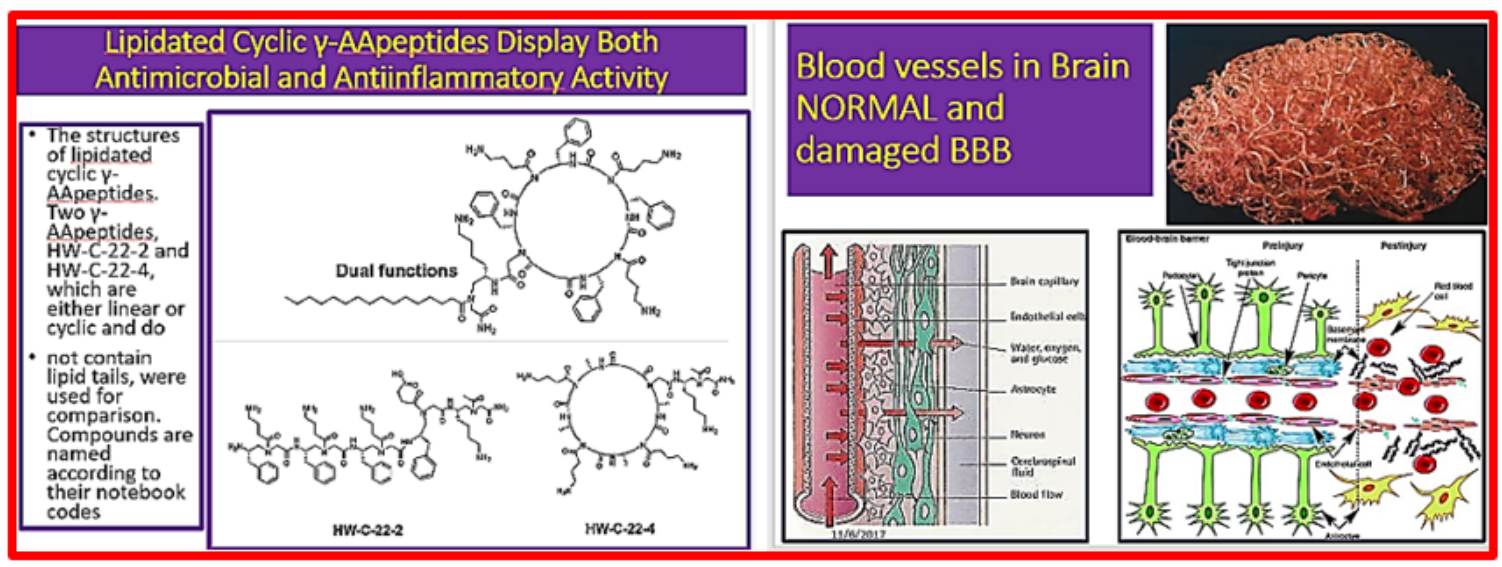

Figure 1

The hallmark brain damage in Parkinson's disease is thought to be the work of a misfolded, rogue protein that spreads from brain cell to brain cell like an infection. Now, researchers have found that the normal form of the protein- $\alpha$-synuclein $(\alpha S)$ - may actually defend the intestines against invaders by marshaling key immune cells. But chronic intestinal infections could ultimately cause Parkinson's, the scientists suggest, if $\alpha \mathrm{S}$ migrates from overloaded nerves in the gut wall to the brain.

"The gut-brain immune axis seems to be on a cusp of an explosion of new insights, and this work offers an exceptionally exciting new hypothesis", says Charles Bevins, an expert in intestinal im- munity at the University of California, Davis, who was not involved with the study.

The normal function of $\alpha \mathrm{S}$ has long been a mystery. Though the protein is known to accumulate in toxic clumps in the brain and the nerves of the gut wall in patients with Parkinson's disease, no one was sure what it did in healthy people. Noting that a region of the $\alpha \mathrm{S}$ molecule behaves similarly to small, microbe-targeting proteins that are part of the body's immune defenses, Michael Zasloff, an immunologist at Georgetown University Medical Center in Washington, D.C., set out to find whether $\alpha \mathrm{S}$, too, might help fend off microbial invaders.
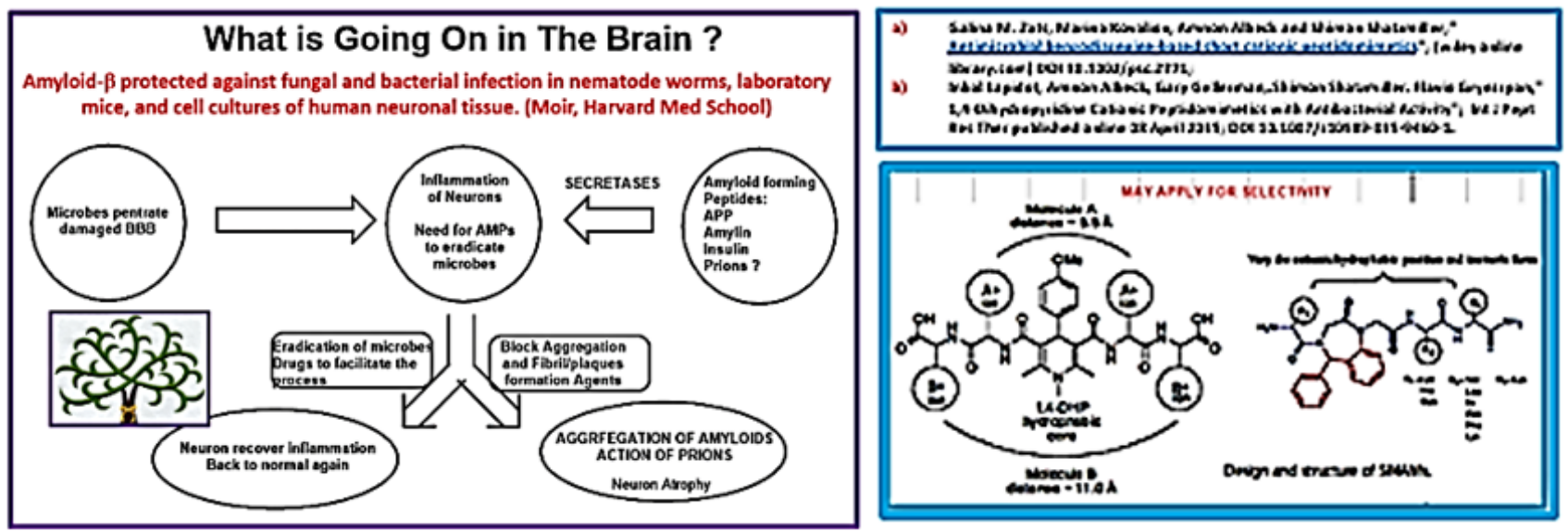

Figure m 
Introducing antimicrobial peptide surrogates to eradicate cerebral microbiome

Although peptides may enter the BBB into the brain from the bloodstream, in bidirectional traffic [47], antimicrobial peptides may be digested already when in the blood due to intensive enzymatic degradation in the serum. It may therefore be more practical to apply surrogates of such antimicrobial peptides that may con- tain scaffolds that cross the BBB and thereby enable eradication of the microbial dwellers of the inner brain.

Recently $[48,49]$ such mimics were prepared and tested for crossing into the mouse brain via the blood system, the agents were injected into the blood stream and detected in the inner brain using confocal microscopy. AIB ( $\alpha$-amino iso-butyric acid as a branched-chain amino acid [50] was used as a "trojan horse" to allow such crossing of the barrier.

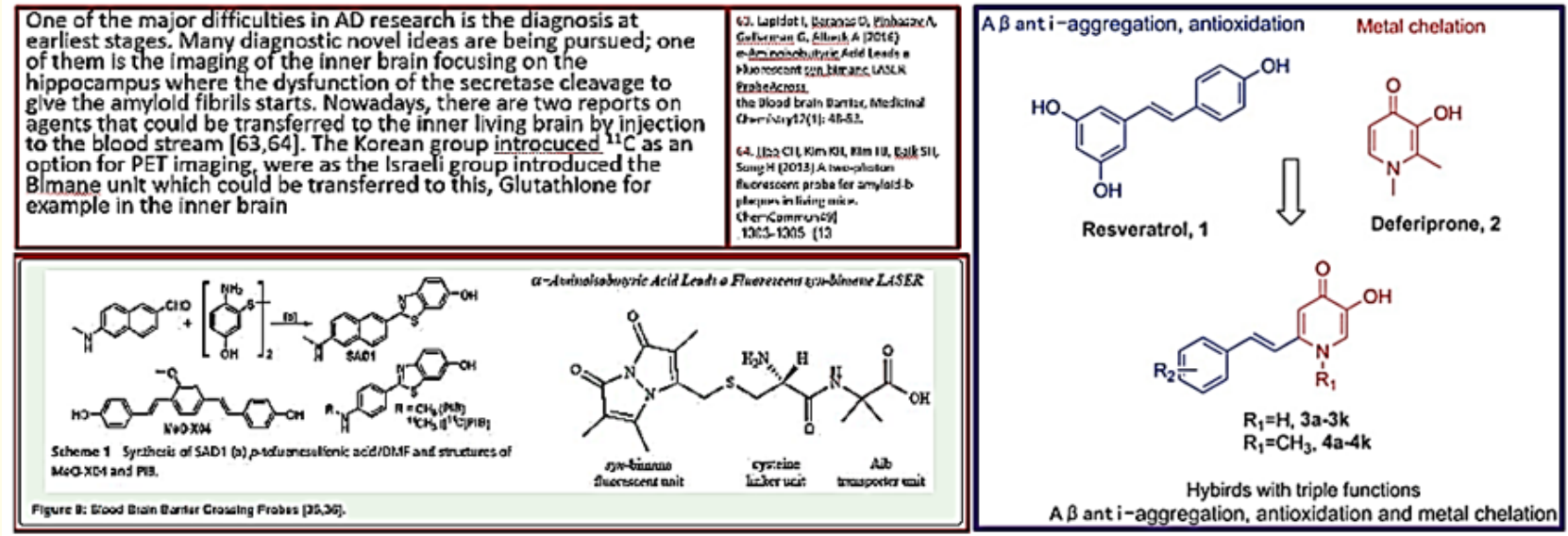

Figure n: BBB crossing agents.

\section{Antioxidants}

While numerous lines of evidence point to increased levels of oxidative stress playing a causal role in several neurodegenerative conditions, our current understanding of the specific role of oxidative stress in the genesis and/or propagation of neurodegenerative diseases remains poorly defined. Even more challenging to the "oxidative stress theory of neurodegeneration" is the fact that many antioxidant-based clinical trials and therapeutic interventions have been largely disappointing in their therapeutic benefit.
It is recommended by physicians and nutritionists to use a "Rainbow" based "Mediterranean diet" as a source of "healthy" antioxidants and vitamins and "Olive Oil [51-53]" which is loaded with bioactive substances. But as a matter of fact, only a few of these can cross the BBB and have a curing effect of neurological dysfunction. Polyphenols are a major agent to treat neurodegeneration [54].

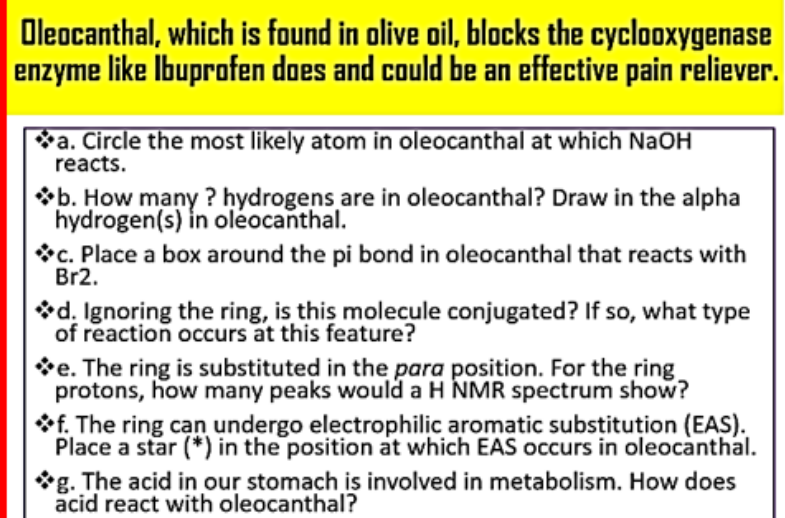

*c. Place a box around the pi bond in oleocanthal that reacts with $\mathrm{Br} 2$.

$\nLeftarrow$ d. Ignoring the ring, is this molecule conjugated? If so, what type of reaction occurs at this feature?

$*$ e. The ring is substituted in the para position. For the ring protons, how many peaks would a H NMR spectrum show?

*f. The ring can undergo electrophilic aromatic substitution (EAS) Place a star $\left({ }^{*}\right)$ in the position at which EAS occurs in oleocanthal. $* \mathrm{~g}$. The acid in our stomach is involved in metabolism. How does acid react with oleocanthal?<smiles>C/C=C(/C=O)[C@H](CC=O)CC(=O)OCCc1ccc(O)cc1</smiles>

Oleocanthal (olive oil)

Figure o: Benefits of Olive Oil. 
Physiological properties of oleocanthal and its putative health benefits Research conducted by Beauchamp and colleagues (Beauchamp., et al. 2005) demonstrated that oleocanthal inhibits cyclooxygenase (COX) enzymes in a dose-dependent manner, mimicking the anti-inflammatory action exerted by ibuprofen. Cyclooxygenase 1 and 2 (COX 1 and COX 2) enzymes are responsible for the conversion of arachidonic acid to Figure Oleocanthal inhibits tau fibrilisation by covalently modifying the PHF- 6 peptide which is crucial for the formation of tau fibrils. The fibrilisation of tau lea- ds to neurofibrillary tangles which are inherently associated with neurodegenerative diseases such as Alzheimer's disease.

Figure Nitric oxide (NO) derived from nitric oxide synthase (NOS), functions as a neurotransmitter and vasodilator, and is important in normal physiological responses. Inducible nitric oxide synthase (iNOS) is a third form of NOS and is not present in resting cells, but rather is induced by inflammatory cytokines. NO produced from iNOS promotes inflammation in chrondocytes and is associated with cartilage degenerative diseases such as 0sreoartritis.
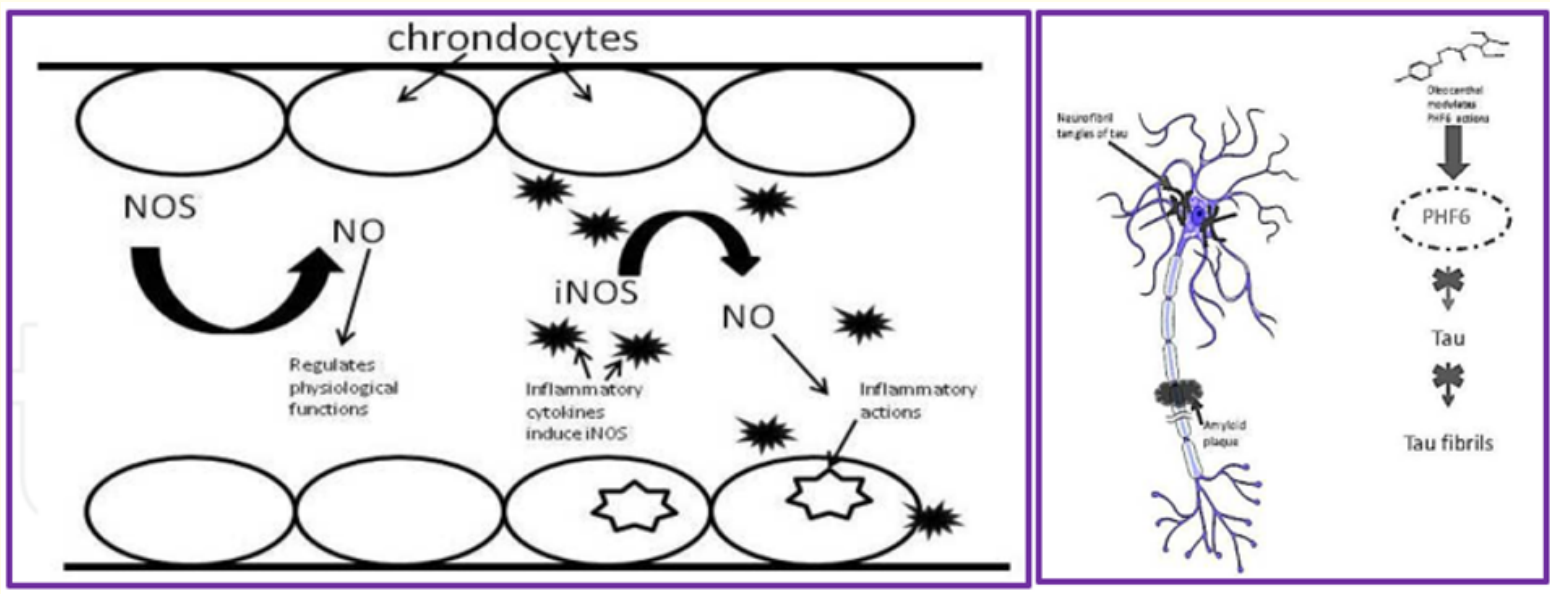

Figure $\mathbf{p}$

Oleocanthal and joint-degenerative disease

In vitro research draws attention to oleocanthal, as a potential therapeutic compound that may be of interest in the quest to find suitable natural NSAIDs for the treatment of joint degenerative disease. Pro-inflammatory cytokines up-regulate the synthesis of cartilage to degrading enzymes, and stimulate nitric oxide (NO) production (Scher., et al. 2007), as well as increase prostaglandin PGE2 production, which have all been implicated in the development of arthritic pain and thus joint-degenerative disease. COX enzymes are a catalyst for the formation of prostaglandins and have also been reported to be highly expressed in arthritic spine in an animal model. Therefore, oleocanthal may influence arthritic pain through inhibition of PGE2 synthesis accompanying COX inhibition. NO plays an integral role in joint-degenerative disease and the stable end product of $\mathrm{NO}$, nitrite $\left(\mathrm{NO}_{2}\right)$, is significantly expressed in arthritic synovial fluid. In osteoarthritis arthritis (OA) pathogenesis, diseased cartilage synthesizes NO spontaneously from diseased chrondocytes (Tung., et al. 2002). NO is biosynthesized by nitric oxide synthase (NOS). Another form of NOS is inducible NOS (iNOS) which is largely responsible for the inflammatory actions of NOS. Iacano and collegues (2010) have shown that oleocanthal and synthesized derivatives, decrease production of iNOS protein expression in LPS challenged murine chondrocytes, dose dependently, further highlighting the anti-inflammatory actions of oleocanthal and the pharmacological potential. Also, as oleocanthal mediates prostaglandin synthesis via inhibitory actions on COX enzymes, it is possible that oleocanthal may exert pharmacological actions in the treatment of both rheumatoid arthritis and osteoarthritis through COX inhibition.

Polyphenols exhibit strong potential to address the etiology of neurological disorders as they attenuate their complex physiology by modulating several therapeutic targets at once. Firstly, we review the advances in the therapeutic role of polyphenols in cell and animal models of AD, PD, MS, and HD and activation of drug targets for controlling pathological manifestations. Secondly, we present principle pathways in which polyphenol intake translates into therapeutic outcomes. In particular, signaling pathways like PPAR, Nrf2, STAT, HIF, and MAPK along with modulation of immune response by polyphenols are discussed. Although current polyphenol researches have limited impact on clinical practice, they have strong evidence and testable hypothesis to contribute clinical advances and drug discovery towards age-related neurological disorders. 

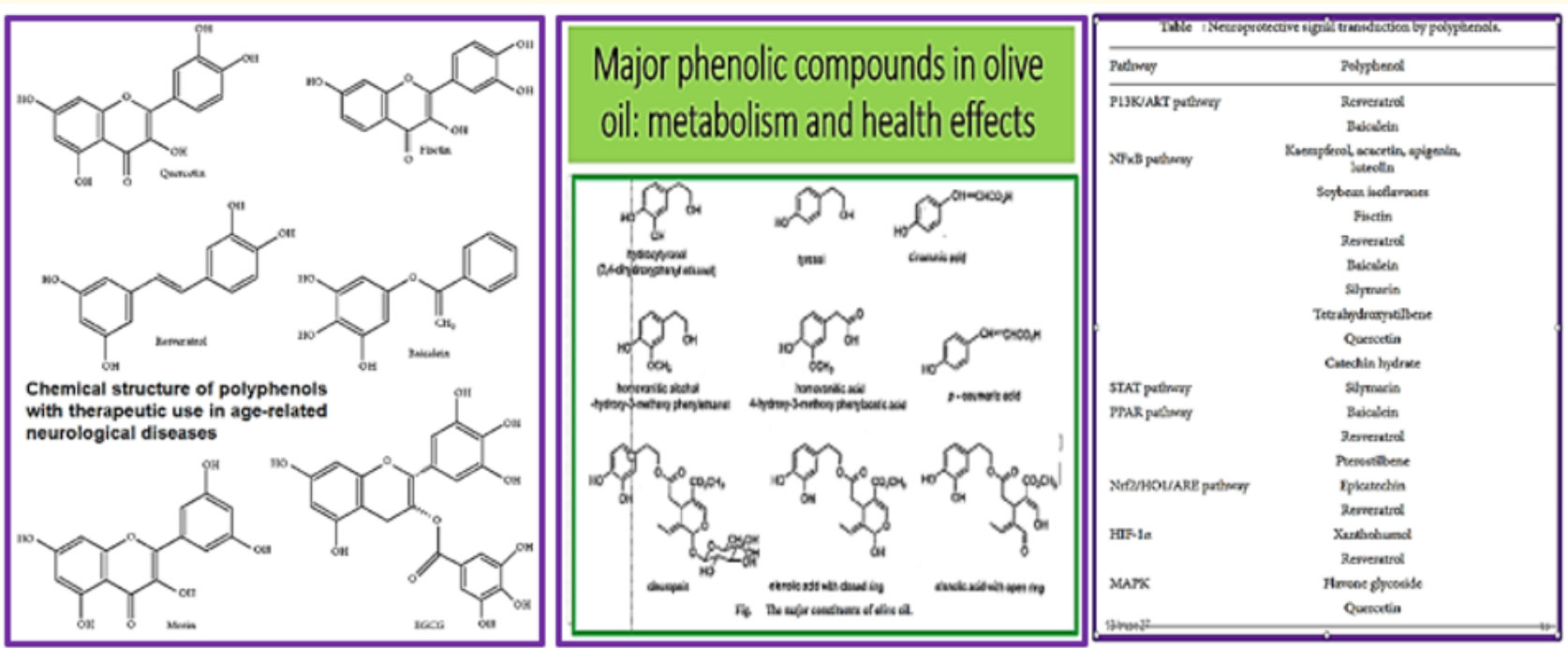

Figure q
Polyphenols also protect mitochondria from pathological events by triggering presurgical cell signaling. Polyphenols increase antioxidant enzymes, that is, catalase, superoxide dismutase (SOD1, SOD2), and PR survival Bcl-2 and PERK pathways. Downregulation of Bad/Bax, c-jun, JNK, COX-2, AP-1, and caspase-3 also contributes to the survival of neurons. Polyphenols also help in improving cognitive abilities by inhibiting AChE and BChE. The inhibition of these enzymes plays an important role in clinical medicine of AD. Apart from their anti-AChE activity, polyphenols also induce metal chelation and modulate autophagy and prion proteins. These features along with the reduction of $A \beta$ toxicity, reduction of neural lesions, and activation of cell survival genes are of particular relevance to neurodegenerative diseases. The activation of the novel spectrum of these molecular targets forms the underlying mechanism of neuroprotection by polyphenols. The lack of toxic effect and availability from natural sources makes polyphenols as clinically relevant therapeutics in neurodegeneration.

Synergic effects in the quest for remedy suitable for neurodegeneration will include antimicrobial peptides and antioxidants to cure inflammation and infection [55,56].

\section{Conclusion}

Research has shown that neuron cells are particularly vulnerable to oxidative damage due to their high polyunsaturated fatty acid content in membranes, high oxygen consumption, and weak antioxidant defense. However, the exact molecular pathogenesis of neurodegeneration related to the disturbance of redox balance remains unclear. Novel antioxidants have shown great potential in mediating disease phenotypes and could be an area of interest for further research. We provide an updated discussion on the roles of ROS in the pathological mechanisms of Alzheimer's disease, Huntington's disease, Parkinson's disease, amyotrophic lateral sclerosis, and spinocerebellar ataxia, as well as a highlight on the antioxidant-based therapies for alleviating disease severity.

\section{Bibliography}

1. https://www.who.int/mediacentre/factsheets/fs310/en/index1.html

2. https://www.cdc.gov/nutrition/micronutrient-

3. ma lnutrition/index.htm l ? C D C _ A A refVal=https $\% 3 \mathrm{~A} \% 2 \mathrm{~F} \% 2 \mathrm{Fww}$

4. w.cdc.gov $\% 2$ Fimmpact $\% 2$ Findex.html

5. Jamison DT., et al. editors. Chapter 44.

6. Jamison DT, Breman JG, Measham AR, et al., editors. Chapter 44.

7. Prevention of Chronic Disease by Means of Diet and Lifestyle Changes Washington (DC): The International Bank for Reconstruction and Development/The World Bank; New York: Oxford University Press; (2006).

8. ChengPeng., et al. "Biology of Ageing and Role of Dietary Antioxidants Hindawi Publishing Corporation". BioMed Research International (2014). 
9. CW Hung., et al. "Ageing and neurodegenerative diseases". Ageing Research Reviews 9.1 (2010): S36-S46.

10. SL Albarracin., et al. "Effects of natural antioxidants in neurodegenerative diseases". Nutritional Neuroscience 15.1 (2012): 1-9.

11. J Lundkvist and J Näslund. " $\gamma$-secretase: a complex target for Alzheimer's disease". Current Opinion in Pharmacology 7.1 (2007): 112-118.

12. Timothy R Sampson., et al. "Gut Microbiota Regulate Motor Deficits and Neuroinflammation in a Model of Parkinson's Disease". Cell 167 (2016): 1469-1480.e12.

13. Wei Wei Chen., et al. " Role of neuroinflammation in neurodegenerative diseases (Review)". Molecular Medicine Reports 13.4 (2016): 3391-3396.

14. Bess Frost and Marc I Diamond. "Prion-like Mechanisms in Neurodegenerative Diseases". Nature Reviews Neuroscience 11.3 (2010): 155-159.

15. https://foodrevolution.org/blog/eating-the-rainbow-healthbenefits/

16. https://www.naturalbalancefoods.co.uk/community/dietary-needs/the-rainbow-diet-meal-plan/

17. https://www.naturallifenews.com/pdf/Rainbow-Diet.pdf

18. Suparna Roy Sarkar and Sugato Banerjee. "Gut microbiota in neurodegenerative disorders". Journal of Neuroimmunology 328 (2019): 98-104.

19. Richard Daneman and Maria Rescigno. "The Gut Immune Barrier and the Blood-Brain Barrier: Are They So Different?". Immunity 31.5 (2009): 722-735.

20. Shimon E Shatzmiller. "Gut Microbes Start Neurodegeneration - The Inflammation Approach". EC Pharmacology and Toxicology SI.01 (2017): 01-03.

21. Caroline S Zhu., et al. "A Review of Traumatic Brain Injury and the Gut Microbiome: Insights into Novel Mechanisms of Secondary Brain Injury and Promising Targets for Neuroprotection". Brain Sciences 8.6 (2018): E113.

22. I Figueira., et al. "Polyphenols journey through blood-brain barrier towards neuronal protection". Scientific Reports (2017): 11456.

23. César G Fraga., et al. "The effects of polyphenols and other bioactives on human health". Food and Function 10 (2019): 514528.
24. Ethan Stolzenberg., et al. "A Role for Neuronal Alpha-Synuclein in Gastrointestinal Immunity". Journal of Innate Immunity 9.5 (2017): 456-463.

25. William A Banks. "Peptides and the blood-brain barrier". Peptides 72 (2015): 16-19.

26. Moshe Gavish., et al. "Enigma of the Peripheral Benzodiazepine Receptor". Pharmacological Reviews 51.4 (1999): 629-650.

27. Shimon Shatzmiller., et al. "Combatting the Microbial Onset of Neurodegeneration the Peptide Surrogate Approach". EC Pharmacology and Toxicology 6.3 (2018): 152-184.

28. GalinaM Zats., et al. "Antimicrobial benzodiazepine-based short cationic peptidomimetics". Journal of Peptide Science 21.6 (2015): 512-519.

29. Inbal Lapidot., et al. "1,4-Dihydropyridine Cationic Peptidomimetics with Antibacterial Activity". International Journal of Peptide Research and Therapeutics 21.3 (2015): 243-247.

30. https://www.theguardian.com/lifeandstyle/2014/jul/12/ ask-a-grown-up-why-are-blueberries-blue

31. Anthony Ananga., et al. "Production of Anthocyanins in Grape Cell Cultures: A Potential Source of Raw Material for Pharmaceutical, Food, and Cosmetic Industries".

32. "10 Proven Health Benefits of Blueberries".

33. Ana Rodriguez-Mateos., et al. "Procyanidin, Anthocyanin, and Chlorogenic Acid Contents of Highbush and Lowbush Blueberries". Journal of Agricultural and Food Chemistry 60.23 (2012): 5772-5778.

34. Ana Rodriguez-Mateos., et al. "Intake and time dependence of blueberry flavonoid-induced improvements in vascular function: a randomized, controlled, double-blind, crossover intervention study with mechanistic insights into biological activity". The American Journal of Clinical Nutrition 98.5 (2013): 1179-1191.

35. Zoriţa Diaconeasa., et al. "Hplc/Pda-Esi/Ms Identification of Phenolic Acids, Flavonol Glycosides and Antioxidant Potential in Blueberry, Blackberry, Raspberries and Cranberries". Journal of Food and Nutrition Research 2.11 (2014): 781-785.

36. Yun JM., et al. "Delphinidin, an anthocyanidin in pigmented fruits and vegetables, induces apoptosis and cell cycle arrest in human colon cancer HCT116 cells". Molecular Carcinogenesis 48.3 (2009): 260-270. 
37. Lock K., et al. "The global burden of disease attributable to low consumption of fruit and vegetables: implications for the global strategy on diet". Bulletin of the World Health Organization 83.2 (2005): 100-108.

38. Laura Este'vez and Ricardo a Mosquera. " Molecular Structure and Antioxidant Properties of Delphinidin". The Journal of Physical Chemistry A 112.42 (2008): 10614-10623.

39. Hertog MGL., et al. Lancet 342 (1993): 1007. /doi. org/10.1016/0140-6736(93)92876-U.

40. Trichopoulou A and Vasilopoulou E. "Mediterranean diet and longevity". British Journal of Nutrition 84 (2000): 205.

41. Silvina Bartesaghi and Rafael Radi. "Fundamentals on the biochemistry of peroxynitrite and protein tyrosine Nitration". Redox Biology 14 (2018): 618-625.

42. https://medicalxpress.com/news/2016-10-inflammationtriggers-unsustainable-immune-response.html

43. Beyer Marc., et al." Tumor-necrosis factor impairs CD4+ T cellmediated immunological control in chronic viral infection". Nature Immunology 17.5 (2016): 593-603.

44. Shatzmiller S., et al. "Blood Brain Barrier Crossing for Therapeutic and Diagnostic Agents". SM Journal of Neurological Disorders and Stroke 2.2 (2016): 1012.

45. https://www.sciencemag.org/news/2018/11/do-gut-bacteria-make-second-home-our-brains

46. https://abstractsonline.com/pp8/\#!/4649/presentation/32057

47. Brian J Spencer and Inder M Verma. " Targeted delivery of proteins across the blood-brain barrier". PNAS 104.18 (2007) 7594-7599.

48. Lapidot I., et al. " $\alpha$-Aminoisobutyric Acid Leads a Fluorescent syn-bimane LASER Probe Across the Blood-brain Barrier". Medicinal Chemistry 12.1 (2016): 48-53.

49. Heo CH., et al. "A two-photon fluorescent probe for amyloidb plaques in living mice". Chemical Communications 49.13 (2013):1303-1305.

50. John D Fernstrom. "Branched-Chain Amino Acids and Brain Function". The Journal of Nutrition 135.6 (2005): 1539S$1546 \mathrm{~S}$.

51. Alaa H Abuznait., et al. "Oxidative stress and cerebral endothelial cells: Regulation of the blood-brain-barrier and antioxidantbased interventions". ACS Chemical Neuroscience 4 (2013): 973-982.
52. Cicerale S., et al. "Oleocanthal: A Naturally Occurring Anti-Inflammatory Agent in Virgin Olive Oil. In Olive Oil - Constituents, Quality, Health Properties and Bioconversions (Dimitrios, B., Ed.) (2012): 357-374. InTech, Rijeka, Croatia.

53. Kellie L Tuck and Peter J Hayball. "Major phenolic compounds in olive oil: metabolism and health effects". Journal of Nutritional Biochemistry 13.11 (2002): 636-644.

54. Khushwant S Bhullar and HP Vasantha Rupasinghe. "Polyphenols: Multipotent Therapeutic Agents in Neurodegenerative Diseases". Oxidative Medicine and Cellular Longevity (2013): ID 891748, 18.

55. Xiaozhe Wu., et al. "Synergistic effects of antimicrobial peptide DP7 combined with antibiotics against multidrug-resistant bacteria". Drug Design, Development and Therapy 11 (2017): 939-946.

56. ZewenLiu., et al. "Oxidative Stress in Neurodegenerative Diseases: From Molecular Mechanisms to Clinical Applications". Oxidative Medicine and Cellular Longevity (2017).

\section{Volume 2 Issue 8 August 2019}

(C) All rights are reserved by Shimon Shatzmiller., et al. 